\title{
Data, information and global change: how long will your data last?
}

\author{
This article is reprinted with permission of the Canadian \\ Global Change Program of the Royal Society of Canada. The \\ author is Co-chairman, Data and Information Systems Panel, \\ Canadian Global Change Program; Data Library Service, \\ University of Toronto. Although originally directed at \\ workers in Canada, the article is of fundamental rele- \\ vance internationally.
}

\section{Introduction}

Data collection is expensive. and the full research potential of good lata is not often realised by the original data collectors or principal investigators. Data can be reanalysed to test new hypotheses, with new statistical techniques, or to provide added insight to comparalive analyses using data from other points in time or geographic areas. Data that are collected with reference to a particular point in ime. furthermore, are irreplaceable, since they can never be replizated. In addition to legal and evidentiary requirements for data preservation, the ethics of the research process demands that the data upon which research is based should be preserved in order to be wailable for replicative analysis. Data in computer-readable form are especially fragile and subject to loss or destruction if not carefully managed from the moment of their collection.

The long-term preservation of computer-readable data involves the management of three discrete components: the data per se, the physical media on which the data files are stored, and the documentation describing the data files. A possible fourth component is the boftware with which the data may have been collected, organised, retrieved, used or analysed.

Datal files may become unusable when the documentation describing their content is lost or destroyed, when the content of a file has been accidentally or erroneously altered, e.g. when a file has been copied over another file or errors have been introduced during editing or analysis, when software with which data are stored becomes obsolete or is no longer upwardly compatible with new versions of the software, when files are accidentally erased, or when the physical media on which the data files are stored are no longer readable. The software industry estimates the life-span of software at approximately 13 years.

The physical media on which data are stored can become unreadable through natural decaly or degradation, through physical acts or accidents which render the media themselves no longer readable or which alter or destroy the magnetic or other properties which comprise the recording, or through the lack of equipment capable of reading the physical medium due to its obsolete format.

In order to ensure the long-term preservation of data, procedures must be implemented to preserve not only the physical media or carricrs, but also the data and the documentation which describe il.

In many instances, it is not feasible for the original data collectors to establish the infrastructure to ensure the long-term preservation of the data, e.g. in the course of short-term research projects. In such cases. arrangements should be made to transfer the data to an existing data archive. A directory of Canadian data archives in uni- versities is available from the Secretariat of the Canadian Global Change Program of The Royal Society of Canada, or from the author.

\section{Recommended procedures}

The following are recommended procedures to ensure the long-term preservation of computer-readable data:

\section{The data}

1 Maintain copies of data in internationally approved data exchange formats, where possible, or in non-software dependent formats (i.e. 'flat' character or binary fields; no 'system' files; no compressed files).

2 Maintain copies of original data prior to the cleaning, recoding, collapsing, or creation of derived variables, and at all stages of data cleaning. Alternately, where applicable, maintain copies of the algorithms that allow recovery of the original data.

3 Where data must be stored in system-dependent formats, main tain documentation of the formats, compression algorithms, name and version of software. Note that 'there are no significant standards in place to ensure future access to compressed data.' (National Archives of Canada Report of Working Group, 1993. p. 11)

4 Where data must be stored in system-dependent formats, migrate to new versions of software as new software is installed. Check the integrity of new format against old format before destroying or discarding data in superseded formats.

5 Store software in the form of source code, with full documentation or commentary, rather than as object code, where possible.

6 Where data are maintained in databases, relational or otherwise, documentation should include a detailed system overview, field specifications of all component database structures, specifications of all field linkages, etc. which comprise the database definition and are needed to reconstruct the database.

\section{The media}

1 Maintain at least two copies of each physical carrier of electronic data (up to five copies if the physical carrier is a microcomputer diskette).

2 Maintain an off-site backup copy of each physical carrier of irreplaceable, irreplicable or otherwise valuable data.

3 Store all physical carriers in an optimum and controlled physical environment. Not all media are as sensitive as magnetic tapes, but the optimum environment for magnetic tapes will suit all other carriers currently known. The environment should be maintained at a constant temperature of $18^{\circ} \mathrm{C}\left(65^{\circ} \mathrm{F}\right)$ and humidity levels of $40 \%$, with fluctuations of no more than $5^{\circ} \mathrm{F}$ or $5 \%$ $\mathrm{RH}$. The air should be clean, free of all dust particles. smoke, etc. Magnetic media should never be stored or placed close to magnets or machines containing electric coils.

4 All magnetic media should be stored vertically, and handled with care. For example, open-reel magnetic tapes should be handled by the hub only, disk-type media should not be bent nor 
placed on rough surfaces, and should be stored in caddies, cases or protective sleeves.

5 When shipping physical carriers, they should be packaged in rigid containers with bubble-type padding, not styrofoam or shredded paper. Packages should be clearly marked 'magnetic media' if applicable.

6 Schedule regular cleaning and rewinding of all reel-type magnetic media, i.e. clean open-reel magnetic tapes (3420s) annually, paying particular attention to state of blade in tape cleaner

7 Schedule regular recopying of all files onto new physical carriers:

$\begin{array}{ll}\text { Medium open reel tapes } & 5 \text { years } \\ 3480 \text { cartridge tapes } & 5 \text { years } \\ \text { DC2000 or DC6000 cassettes } & 1-2 \text { years } \\ \text { DAT tapes } & 1-2 \text { years } \\ \text { Exabyte tapes } & 2 \text { years } \\ \text { magnetic (floppy) disks } & 1 \text { year } \\ \text { optical disks } & 10 \text { years (?) }\end{array}$

8 Migrate files to new storage media when new media become standardised and before devices to read old media are no longer available.

\section{The documentation}

1 Collect complete documentation as soon as possible, preferably while the principal investigator is available. Documentation should be sufficiently complete as to allow another user to utilise the data without recourse to the original collector of the data, and should include:

- statement of objectives of data collection project,

- detailed description of data collection, including methodology, description of universe and sampling frame, etc.,

- description of pretest activities, where applicable,

- copies or technical specifications of data collection instruments (e.g. questionnaires, software. or hardware), as applicable,

- instructions to interviewers (if a personal interview) or other instructions to survey respondents, where applicable,

- description of post-processing, data cleaning, anonymisation, data conversion algorithms, etc..

- description of weights and procedures for calculating weights, where applicable,

- detailed description of order, size, format, and content and/or values of all variables or field, including beginning and ending field tags or other field delimiters, where applicable.

- detailed descriptions of derived variables, including source algorithms,

- assessment of data quality, including restrictions on applicability, if any.

2 Where documentation is computer-readable, maintain it in a non-system dependent format(s) where possible.

3 Maintain backup copies of all documentation, including off-site backup copies of all documentation of irreplaceable, non replicable, or valuable data.

4 Update documentation as data are changed in the process of post-processing, analysis, etc.

\section{Selected bibliography:}

American Physical Society, 1991, Report of the APS Task Force on Electronic Information Systems: Bulletin of the American Physical Society, v. 36, no. 4. pp. $1119-1151$

Balon, B J and Gardner. H W, 1988, Disaster planning for electronic records: Records Management Quarterly v. 22 no. 3, pp. 20-25.
Boston, G, ed., 1992. Archiving the audio-visual heritage. Joint Technical Symposium (3rd), 1992, Ottawa. Ontario: Milton Keynes, UNESCO

Day, M W, 1990, Preservation problems of electronic text and data.: East Midlands Branch of the Library Association, Loughborough,UK. Occit sional Paper 3.

DeWhitt, B L, 1987, L cong-term preservation of data on computer magnetic media part I [andl II: Conservation Administration News, April 1987 [and] July 1987.

Geller, S B, 1983, Care and handling of computer magnetic storage media National Bureau of Standards Special Publication 500-101. Washington. DC.

Lesk, M, 1992, Preservation of new technology, a report of the Technology Assessment Advisory Committee to the Commission on Preservation and Access. Washington. DC.

Mohlhenrich, J, ed.. Preservation of electronic formats and electronic formats for preservation: Fort Atkinson. Wisconsin, Highsmith Press. 1993.

National Archives of Canada.. 1989. Proceedings of Symposium on Conservation in Archives, Ottawa, Ontario, May 1988

National Archives of Canada, 1993, Report of the Working Group on Conservation Standards and Technologies.

National Research Council. Commission on Engineering and Technical Systems, National Materials Advisory Board and Committee on Preservation of Historical Records, 1986. Preservation of historical records: Washington, DC, National Academic Press.

Saffady, W, 1991, Stability, care and handling of microforms, magnctic media and optical disks. Library technology reports 27, pp. 5-117

United Nations Advisory Committee for the Co-ordination of Intormation Systems (ACCIS), 1992, Information system security guidelines for the United Nations organizations: New York. NY, United Nations.

US Congress House of Representatives, Committee on Ciovernment Operittions, 1990, Taking at byte out of history: the archival preservation of federal computer records. 101st Congress. 2nd Session, H R 987.

Weir, T E, 1993, 3480 class tape cartridge drives and archival tape storage: technology assessment report: National Archives lechnical information paper, 4, Springfield, Viginia, National Technicil Information Service.

Williamson. M P. 1991. The 3480) type tape cartridge: potential data storage risks, and care and handling procedures to minimize risks: National Institute of Standards and Technology Special Publication 500-199. Washington, $\mathrm{DC}$.

This article was originally printed as the first in a series of brochures (ISSN 1198-5755), published by the Canadian Clobal Change Program of The Roval Society of Canada, on issues of concern related to data and information for global change research and decision-making. For additional information on this series or to obtain more information on the CGCP, please contact:

Dave Henderson, Canadian Global Change Program. ch The Royal Society of Canada,

PO Box 9734. Ottawa.

Ontario KIG 5.44 ;

Telephone: (613) 991-.5640: Fax: (613) 991-6996;

Internet: dhenders@rsc.ca:

Internet gopher: datalib.library.ualberta.ca 70 ;

WEB: cgcprse

The Canadian Global Change Program ( $C G C P$ ) is the national focus for global change information. edacation and research activity in Canada. Its activities are closely linked to those of The Royal Society of Canada, which established the CGCP in 1985. The $C G C P$ is a conduit to other national and international efforts, including the International Geosphere-Biosphere Programme, the International Human Dimensions Programme and the World Climate Research Program (through the Canadian Climate Program).

Core funding for the $C G C P$ is provided by the federal government through Environment Canada's Green Plan. Additional funds and other support are provided by the Richard lvey Foundation, federal research agencies, provincial governments and agencies, the private sector and non-governmental organisations. 